\title{
STUDY OF SOME BIOLOGICAL PARAMETERS OF THE RED CALIFORNIAN EARTHWORM EISENIA FOETIDA (SAVIGNY, 1826) IN VERMICOMPOST FOLLOWING THE APPLICATION OF WOOD VINEGAR
}

\author{
Koç, İ. \\ Bitlis Eren University, Faculty of Engineering and Architecture, Department of Environmental \\ Engineering, 13000, Bitlis, Turkey \\ (e-mail: ibrahimkoc47@gmail.com; phone: +90-434-228-0075; fax: +90-434-222-0101)
}

(Received 28 ${ }^{\text {th }}$ Jan 2019; accepted $27^{\text {th }}$ Feb 2019)

\begin{abstract}
This study was carried out with the purpose to assess some biological parameters of the red Californian worm (Eisenia foetida) in vermicompost, to which two types of wood vinegar, manufactured from poultry manure (A) and nutshell (B), have been applied. The study was implemented under laboratory conditions with Coincidence Parcels Trial Layout and 5 repetitions in 2018. Profile Analysis Technique was used in the in the investigation of wood vinegar types and the effect of various doses on the weight gain and number of worms on a weekly basis, while Factorial ANOVA was used in the investigation of their effects on cocoon numbers. The Profile Analysis proved that the interaction between Week $\times$ Dose $\times$ Vinegar type was important in regards to both the weight and number of worms $(\mathrm{P}=$ 0.00). The Factorial ANOVA carried out to investigate the effects of the vinegar type and doses on the cocoon number showed that the Dose $\times$ Vinegar type interaction was important $(P=0.00)$. Therefore, the effects of the vinegar type on the number and weight of worms differed depending on the dosage. In general, it was noted that 3\%- and 5\% dosages of vinegars had positive effects on Eisenia foetida, while the dosage of $100 \%$ had toxic effects. Based on the findings, it is possible that wood vinegars have a potential use with various purposes including bio-pesticides or worm manure.
\end{abstract}

Keywords: agricultural, bio-economy, bio-pesticides, cocoon, pyrolysis liquids, worm

\section{Introduction}

Although earthworms are not abundant in number, they are probably significant factors for sustaining soil productivity in various ways (Edwards and Bohlen, 1992). These living beings are accepted as important indicators of chemical toxicity in the soil ecosystem (Bustos-Obregón and Goicochea, 2002; Yasmin and D'Souza, 2010). Wood vinegar (WV) is a liquid produced through carbonization (pyrolysis liquids) that has been found to had been produced as early as the time of the Neanderthals (Tiilikkala et al., 2010; Cai et al., 2012). Kim et al. (2008) stated with reference to Jang that WV is a clear liquid containing $80-90 \%$ water with the rest being water-soluble organic compounds exceeding 200 in number. Jothityangkoon et al. (2008) stated that the main components of WV were acetic acid paired with organic acids and phenolic compounds, alkanes, alcohols and esters. This product is used as manure, insect repellant or organic fungicide in agriculture. Furthermore, there are studies showing that WV has effects that improve the soil and promote plant growth. As examples of such studies, Jothityangkoon et al. (2008), Yin (2008), Baimark and Niamsa (2009), Velmurugan et al. (2009), Chalermsan and Peerapan (2009), Oramahi and Yoshimura (2013), Hagner (2013), Naml et al. (2014), Koc et al. (2017), De Souza Araújo et al. (2018) and Oramahi et al. (2018) have performed studies concerning the antifungal activities of WV. Studies of Yatagai et al. (2002), Oramahi and Yoshimura (2013) are notable on the issue of anti-termite effects of WV. It was stated by the studies of Shi (2003), 
Jothityangkoon et al. (2008), Yin (2008), Rakmai (2009), Cai et al. (2012) that WV affects plant growth. Regarding the antibacterial effectiveness of WV, positive remarks of Chalermsan and Peerapan (2009), Eric et al. (2012), Duan et al. (2016), Yang et al. (2016), Chukeatirote et al. (2018) and De Souza Araújo et al. (2018) can be noted. Koç et al. (2018) have found significant relations between treatments with WV and nematodes in the soil. Shi (2003) and Rakmai (2009) have obtained findings indicating the activities of $\mathrm{WV}$ that increase the edaphon and the number of bacteria in the root area of plants. There are also studies by Inoue et al. (2000), Yatagai et al. (2002), Jothityangkoon et al. (2008), Diba et al. (2009), Kiarie-Makara et al. (2010), Pangnakorn et al. (2011), Wititsiri (2011), Hagner (2013), Oramahi and Yoshimura (2013) explaining the insecticide or insect-repellant effects of WV. Cai et al. (2012) have reported that WV promotes the development of reactive oxidative species (ROS)sensitive mutant worms, the lifetime of worms is prolonged and their body size increased. $\mathrm{Mu}$ et al. (2003) have stated that WV is a good resource for organic agriculture and is widely used in Japan, both in agriculture and daily life. Kim et al. (2008) have stated that the annual production of WV is around 14.000 tons. Hagner (2013) has stated in his study that there is little information on the toxic effects of WV, while Rakmai (2009) stated referring to Ikeshima that this substance has no toxic effects on humans or animals. Tiilikkala et al. (2010) have reported that pyrolysis liquids have the potential of replacing pesticides and synthetic chemicals in biocide form in the future, and this is based on bio-economic knowledge and depends on its development. As seen in the studies cited above, is it clear that WV has multiple effects.

This study has been carried with the purpose of determining some biological parameters of the red Californian earthworm (Eisenia foetida) in vermicompost after application of two types of wood vinegar obtained from poultry manure (A) and nutshell (B).

\section{Materials and methods}

This study was carried out under laboratory conditions in 2018. The vermicompost required for the experiments was obtained from $100 \%$ cow manure, and cocoons were obtained through reproduction from stock cultures of Eisenia foetida individuals. Wood vinegar (WV), was obtained from a firm developing WV products from poultry manure (A) and nutshell (B) using a gasification machine and bio-coal (Namlı et al., 2014). Vinegar obtain from nutshell (B) is light brown in color, and is characterized by a burning smell. Vinegar obtained from poultry manure (A) however, is darker in color as compared to vinegar $\mathrm{B}$, is more consistent and has a stronger odor. The study was implemented using the Coincidence Parcels Trial Layout with 5 repetitions (Fig. 1). After placing $100 \mathrm{~g}$ vermicompost in $300 \mathrm{~cm}^{3}$ plastic containers, the solution was treated with WV using a $10-\mathrm{mL}$ injector. One percent, $3 \%, 5 \%, 10 \%, 25 \%$ and $100 \%$ WV doses were used in the treatments, and only tap water was injected into the control group. WV treatment was performed only once. Following these procedures, 10 cocoons were placed in each container. The ambient temperature of the study was kept between 20 and $29^{\circ} \mathrm{C}$ and the humidity was kept between 70 and 90\% (Gunadi et al., 2002). With the purpose of maintaining the ambient humidity, $10 \mathrm{~mL}$ tap water was administered to the samples periodically with 2 day intervals. Live weight, number of cocoons laid, and the number of young individuals coming out of cocoons were determined weekly until the young individuals laid cocoons themselves. Profile 
Analysis was used during the investigation of the effects of different doses and vinegar types on weekly body weight gain and number of worms (Mendeș et al., 2007). Factorial ANOVA was used to investigate the effects of the factors mentioned above on the number of cocoons, and Tukey's Multiple Comparison Test was used in determining the different groups (Winer et al., 1991; Mendeş, 2012). The abovementioned analyses were carried out using the SPSS (Ver. 21.0) statistical package programs.

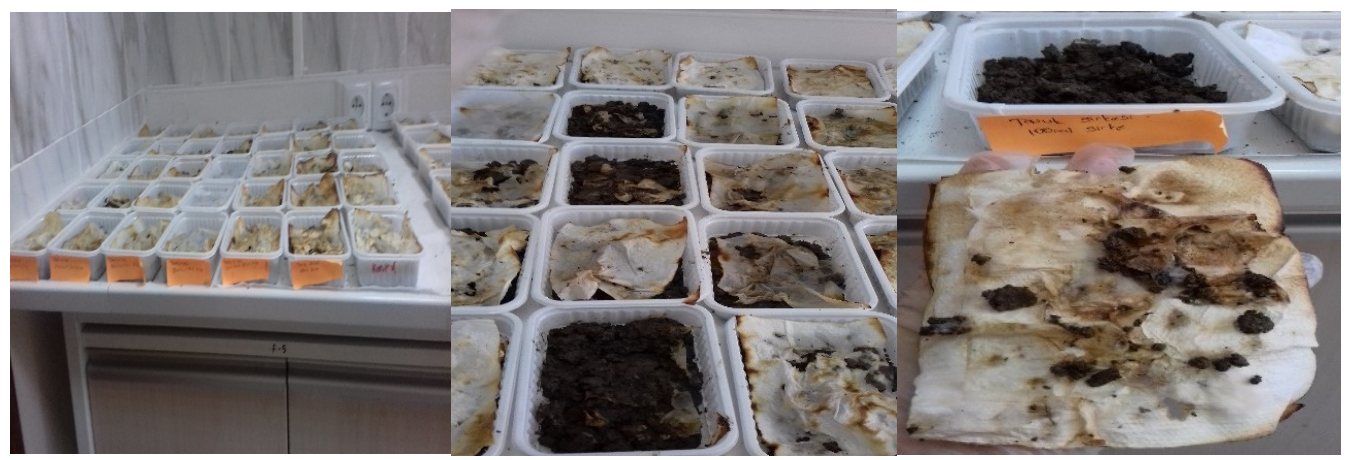

Figure 1. Photo of the experimental culture

\section{Results}

\section{Findings related to the weights of worms}

Profile Analysis results for WV(A) and WV(B) are presented in Figures 2 and 3, respectively. Results of Profile Analysis indicated that the Week $\times$ Dose $\times$ Vinegar type interaction is significant (Wilks' Lambda $=0.253 ; \mathrm{F}=3.61 ; \mathrm{P}=0.00$ ). Meaning that the effect of vinegar type on weekly body weight gain of worms varied based on the doses (Figs. 2 and 3; Table 1). When only week 5 is taken into consideration in comparison with the controls, treatments with WV (B) have not resulted in much difference between the body weights of worms (Fig. 3; Table 1). However, treatments with WV (A) $1 \%$, $25 \%$ and $100 \%$ had negative effects while treatments with $3 \%$ and $5 \%$ had positive effects (Fig. 2; Table 1).

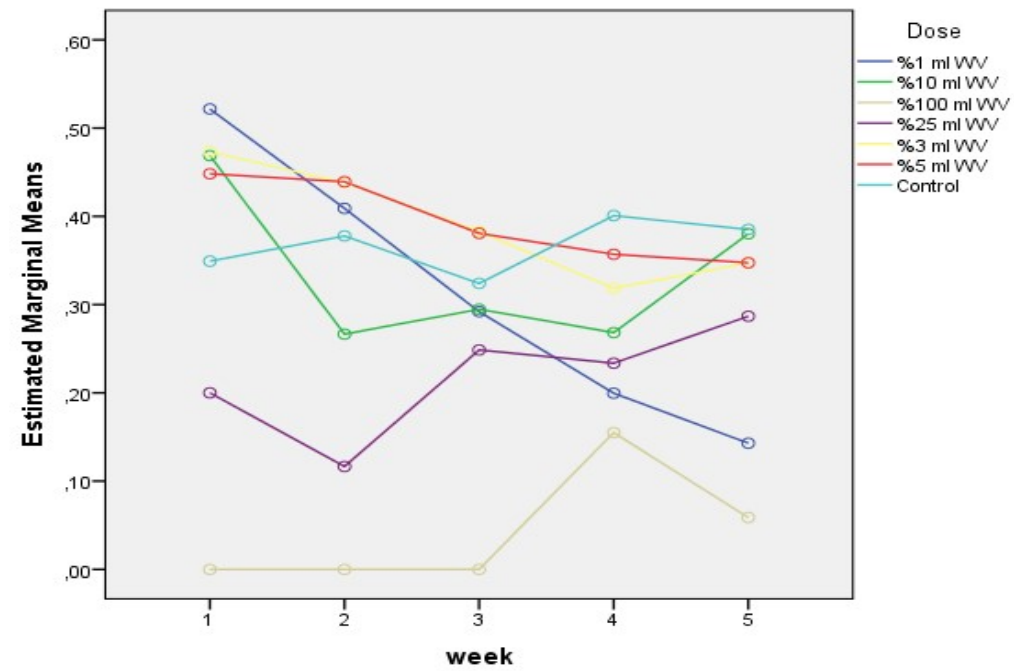

Figure 2. Profile analysis for investigating the effect of WV(A) on weekly body weight gain of worms based on doses 


$$
-4530-
$$

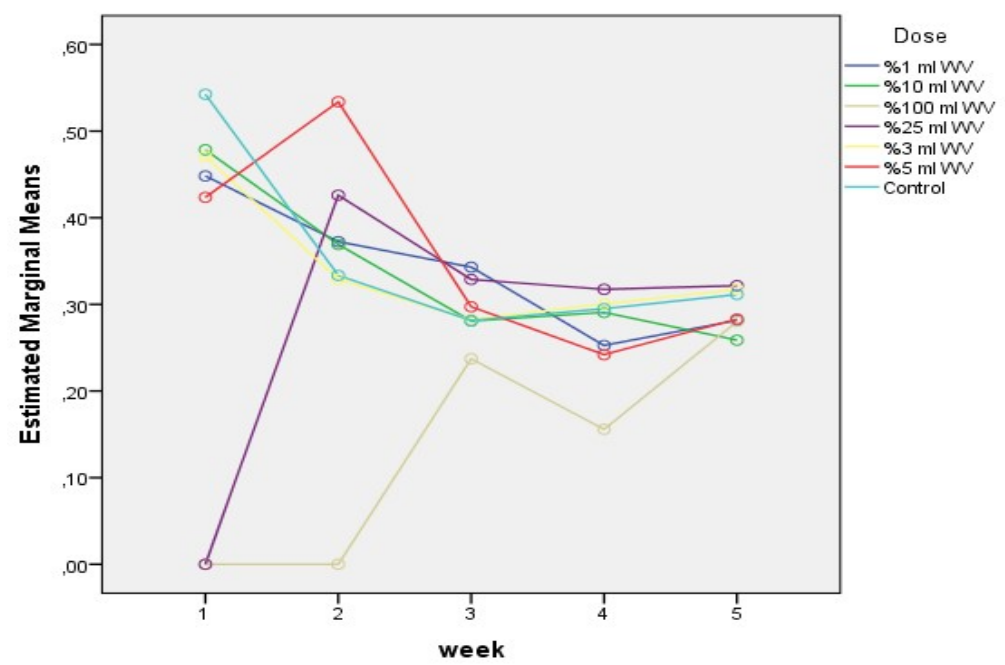

Figure 3. Profile analysis for investigating the effect of $W V(B)$ on weekly body weight gain of worms based on doses

Table 1. Descriptive statistics for the body weight of worms

\begin{tabular}{|c|c|c|c|c|c|c|c|c|c|c|c|}
\hline Week & WV dose & $\begin{array}{c}\text { Vinegar } \\
\text { type }\end{array}$ & Mean & $\begin{array}{c}\text { Std. } \\
\text { deviation }\end{array}$ & $\mathbf{N}$ & Week & WV Dose & $\begin{array}{c}\text { Vinegar } \\
\text { type }\end{array}$ & Mean & $\begin{array}{c}\text { Std. } \\
\text { deviation }\end{array}$ & $\mathbf{N}$ \\
\hline \multirow{24}{*}{$\tilde{\beta}$} & \multirow{3}{*}{$1 \%$} & A & 0.522 & 0.131 & 5 & & \multirow{3}{*}{$1 \%$} & A & 0.409 & 0.076 & 5 \\
\hline & & B & 0.448 & 0.134 & 5 & & & B & 0.372 & 0.114 & 5 \\
\hline & & Total & 0.485 & 0.131 & 10 & & & Total & 0.390 & 0.093 & 10 \\
\hline & \multirow{3}{*}{$3 \%$} & A & 0.473 & 0.043 & 5 & & \multirow{3}{*}{$3 \%$} & A & 0.438 & 0.145 & 5 \\
\hline & & B & 0.471 & 0.112 & 5 & & & B & 0.329 & 0.120 & 5 \\
\hline & & Total & 0.472 & 0.080 & 10 & & & Total & 0.384 & 0.138 & 10 \\
\hline & \multirow{3}{*}{$5 \%$} & A & 0.448 & 0.024 & 5 & & \multirow{3}{*}{$5 \%$} & A & 0.439 & 0.055 & 5 \\
\hline & & B & 0.424 & 0.050 & 5 & & & B & 0.534 & 0.072 & 5 \\
\hline & & Total & 0.436 & 0.039 & 10 & & & Total & 0.487 & 0.078 & 10 \\
\hline & \multirow{3}{*}{$10 \%$} & A & 0.469 & 0.625 & 4 & & \multirow{3}{*}{$10 \%$} & A & 0.267 & 0.309 & 4 \\
\hline & & $\mathrm{B}$ & 0.479 & 0.099 & 5 & & & $\mathrm{~B}$ & 0.369 & 0.036 & 5 \\
\hline & & Total & 0.474 & 0.080 & 9 & 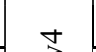 & & Total & 0.324 & 0.198 & 9 \\
\hline & \multirow{3}{*}{$25 \%$} & A & 0.200 & 0.187 & 5 & $\leqslant$ & \multirow{3}{*}{$25 \%$} & A & 0.117 & 0.162 & 5 \\
\hline & & $\mathrm{B}$ & 0.000 & 0.000 & 5 & & & $\mathrm{~B}$ & 0.426 & 0.042 & 5 \\
\hline & & Total & 0.100 & 0.163 & 10 & & & Total & 0.271 & 0.198 & 10 \\
\hline & \multirow{3}{*}{$100 \%$} & $\mathrm{~A}$ & 0.000 & 0.000 & 4 & & \multirow{3}{*}{$100 \%$} & A & 0.000 & 0.000 & 4 \\
\hline & & $\mathrm{B}$ & 0.000 & 0.000 & 5 & & & $\mathrm{~B}$ & 0.000 & 0.000 & 5 \\
\hline & & Total & 0.000 & 0.000 & 9 & & & Total & 0.000 & 0.000 & 9 \\
\hline & \multirow{3}{*}{ Control } & A & 0.349 & 0.058 & 5 & & \multirow{3}{*}{ Control } & A & 0.378 & 0.128 & 5 \\
\hline & & $\mathrm{B}$ & 0.543 & 0.081 & 5 & & & $\mathrm{~B}$ & 0.333 & 0.059 & 5 \\
\hline & & Total & 0.446 & 0.122 & 10 & & & Total & 0.356 & 0.097 & 10 \\
\hline & \multirow{3}{*}{ Total } & $\mathrm{A}$ & 0.359 & 0.191 & 33 & & \multirow{3}{*}{ Total } & $\mathrm{A}$ & 0.302 & 0.209 & 33 \\
\hline & & $\mathrm{B}$ & 0.338 & 0.232 & 35 & & & $\mathrm{~B}$ & 0.338 & 0.169 & 35 \\
\hline & & Total & 0.348 & 0.212 & 68 & & & Total & 0.320 & 0.189 & 68 \\
\hline \multirow{3}{*}{ 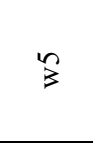 } & \multirow{3}{*}{$1 \%$} & $\mathrm{~A}$ & 0.292 & 0.175 & 5 & \multirow{3}{*}{$\vdots$} & \multirow{3}{*}{$1 \%$} & $\mathrm{~A}$ & 0.200 & 0.274 & 5 \\
\hline & & $\mathrm{B}$ & 0.343 & 0.049 & 5 & & & $\mathrm{~B}$ & 0.253 & 0.025 & 5 \\
\hline & & Total & 0.318 & 0.124 & 10 & & & Total & 0.226 & 0.185 & 10 \\
\hline
\end{tabular}




\begin{tabular}{|c|c|c|c|c|c|c|c|c|c|c|c|}
\hline & \multirow{3}{*}{$3 \%$} & $\mathrm{~A}$ & 0.383 & 0.063 & 5 & & \multirow{3}{*}{$3 \%$} & $\mathrm{~A}$ & 0.318 & 0.035 & 5 \\
\hline & & $\mathrm{B}$ & 0.282 & 0.050 & 5 & & & $\mathrm{~B}$ & 0.301 & 0.043 & 5 \\
\hline & & Total & 0.333 & 0.075 & 10 & & & Total & 0.309 & 0.038 & 10 \\
\hline & \multirow{3}{*}{$5 \%$} & $\mathrm{~A}$ & 0.381 & 0.080 & 5 & & \multirow{3}{*}{$5 \%$} & $\mathrm{~A}$ & 0.357 & 0.024 & 5 \\
\hline & & $\mathrm{B}$ & 0.297 & 0.019 & 5 & & & $\mathrm{~B}$ & 0.242 & 0.027 & 5 \\
\hline & & Total & 0.339 & 0.070 & 10 & & & Total & 0.300 & 0.065 & 10 \\
\hline & \multirow{3}{*}{$10 \%$} & $\mathrm{~A}$ & 0.295 & 0.057 & 4 & & \multirow{3}{*}{$10 \%$} & $\mathrm{~A}$ & 0.268 & 0.179 & 4 \\
\hline & & $\mathrm{B}$ & 0.281 & 0.023 & 5 & & & $\mathrm{~B}$ & 0.291 & 0.034 & 5 \\
\hline & & Total & 0.287 & 0.039 & 9 & & & Total & 0.281 & 0.113 & 9 \\
\hline & \multirow{3}{*}{$25 \%$} & $\mathrm{~A}$ & 0.249 & 0.172 & 5 & & \multirow{3}{*}{$25 \%$} & $\mathrm{~A}$ & 0.234 & 0.136 & 5 \\
\hline & & $\mathrm{B}$ & 0.329 & 0.077 & 5 & & & B & 0.317 & 0.059 & 5 \\
\hline & & Total & 0.289 & 0.133 & 10 & & & Total & 0.276 & 0.108 & 10 \\
\hline & \multirow{3}{*}{$100 \%$} & $\mathrm{~A}$ & 0.000 & 0.000 & 4 & & \multirow{3}{*}{$100 \%$} & A & 0.155 & 0.232 & 4 \\
\hline & & $\mathrm{B}$ & 0.237 & 0.154 & 5 & & & $\mathrm{~B}$ & 0.156 & 0.213 & 5 \\
\hline & & Total & 0.132 & 0.166 & 9 & & & Total & 0.155 & 0.207 & 9 \\
\hline & \multirow{3}{*}{ Control } & $\mathrm{A}$ & 0.324 & 0.075 & 5 & & \multirow{3}{*}{ Control } & $\mathrm{A}$ & 0.401 & 0.034 & 5 \\
\hline & & $\mathrm{B}$ & 0.281 & 0.049 & 5 & & & $\mathrm{~B}$ & 0.295 & 0.021 & 5 \\
\hline & & Total & 0.303 & 0.064 & 10 & & & Total & 0.348 & 0.062 & 10 \\
\hline & \multirow{3}{*}{ Total } & $\mathrm{A}$ & 0.282 & 0.153 & 33 & & \multirow{3}{*}{ Total } & $\mathrm{A}$ & 0.280 & 0.164 & 33 \\
\hline & & $\mathrm{B}$ & 0.293 & 0.074 & 35 & & & $\mathrm{~B}$ & 0.265 & 0.095 & 35 \\
\hline & & Total & 0.288 & 0.118 & 68 & & & Total & 0.272 & 0.132 & 68 \\
\hline \multirow{12}{*}{$\hat{3}$} & \multirow{3}{*}{$1 \%$} & $\mathrm{~A}$ & 0.143 & 0.198 & 5 & & \multirow{3}{*}{$25 \%$} & $\mathrm{~A}$ & 0.287 & 0.055 & 5 \\
\hline & & $\mathrm{B}$ & 0.282 & 0.022 & 5 & & & $\mathrm{~B}$ & 0.322 & 0.059 & 5 \\
\hline & & Total & 0.212 & 0.152 & 10 & & & Total & 0.304 & 0.057 & 10 \\
\hline & \multirow{3}{*}{$3 \%$} & $\mathrm{~A}$ & 0.347 & 0.066 & 5 & & \multirow{3}{*}{$100 \%$} & $\mathrm{~A}$ & 0.059 & 0.117 & 4 \\
\hline & & $\mathrm{B}$ & 0.317 & 0.016 & 5 & & & $\mathrm{~B}$ & 0.280 & 0.256 & 5 \\
\hline & & Total & 0.332 & 0.048 & 10 & & & Total & 0.182 & 0.227 & 9 \\
\hline & \multirow{3}{*}{$5 \%$} & $\mathrm{~A}$ & 0.347 & 0.021 & 5 & 3 & \multirow{3}{*}{ Control } & $\mathrm{A}$ & 0.385 & 0.016 & 5 \\
\hline & & $\mathrm{B}$ & 0.283 & 0.018 & 5 & & & $\mathrm{~B}$ & 0.311 & 0.034 & 5 \\
\hline & & Total & 0.315 & 0.039 & 10 & & & Total & 0.348 & 0.046 & 10 \\
\hline & \multirow{3}{*}{$10 \%$} & $\mathrm{~A}$ & 0.380 & 0.023 & 4 & & \multirow{3}{*}{ Total } & $\mathrm{A}$ & 0.282 & 0.144 & 33 \\
\hline & & $\mathrm{B}$ & 0.259 & 0.033 & 5 & & & $\mathrm{~B}$ & 0.293 & 0.095 & 35 \\
\hline & & Total & 0.313 & 0.070 & 9 & & & Total & 0.288 & 0.120 & 68 \\
\hline
\end{tabular}

\section{Findings on the number of worms}

Profile Analysis results for WV(A) and WV(B) are presented in Figures 4 and 5, respectively. Results of Profile Analysis indicated that the Week $\times$ Dose $\times$ Vinegar type interaction is significant (Wilks' Lambda $=0.05 ; \mathrm{F}=7.83 ; \mathrm{P}=0.00$ ). Meaning that the effect of vinegar type on weekly number of worms varied based on the doses (Figs. 4 and 5, Table 2).

It can be noted that treatments with $1 \%, 25 \%$ and $100 \% \mathrm{WV}$ (A) have negative effects on the number of worms in comparison with controls, while $3 \%$ and $5 \%$ doses have positive effects (Fig. 4; Table 2). It was also seen that the 100\% dose of WV (B) had negative effects in comparison with the controls, while treatments with $3 \%, 5 \%$ and 10\% WV had positive effects (Fig. 5; Table 2). 


$$
-4532-
$$

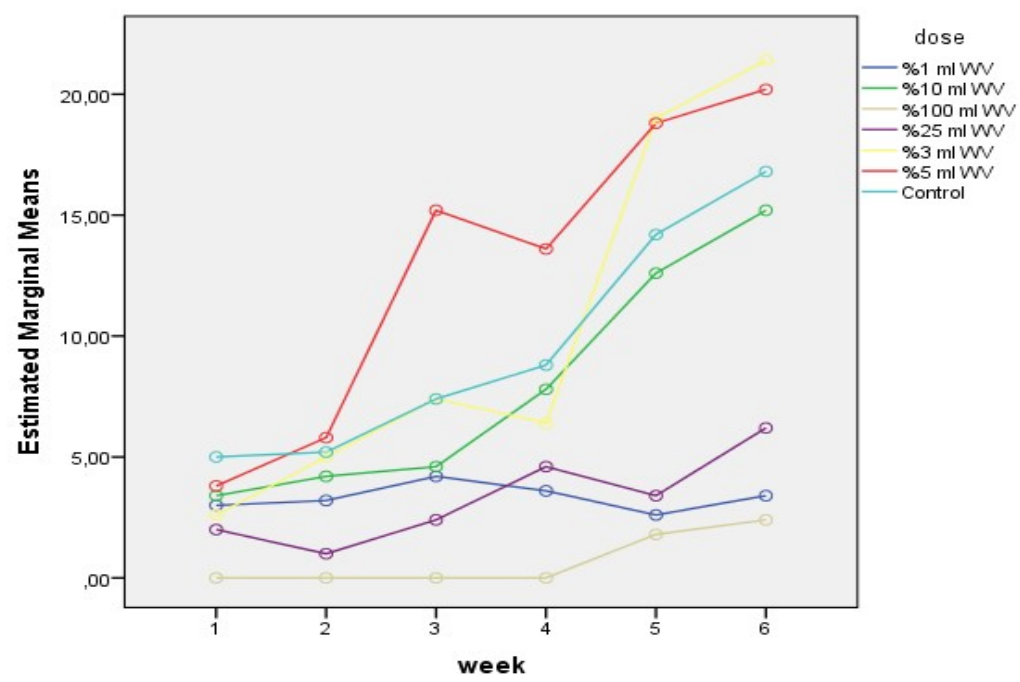

Figure 4. Profile analysis for investigating the effect of $W V(A)$ on weekly number of worms based on doses

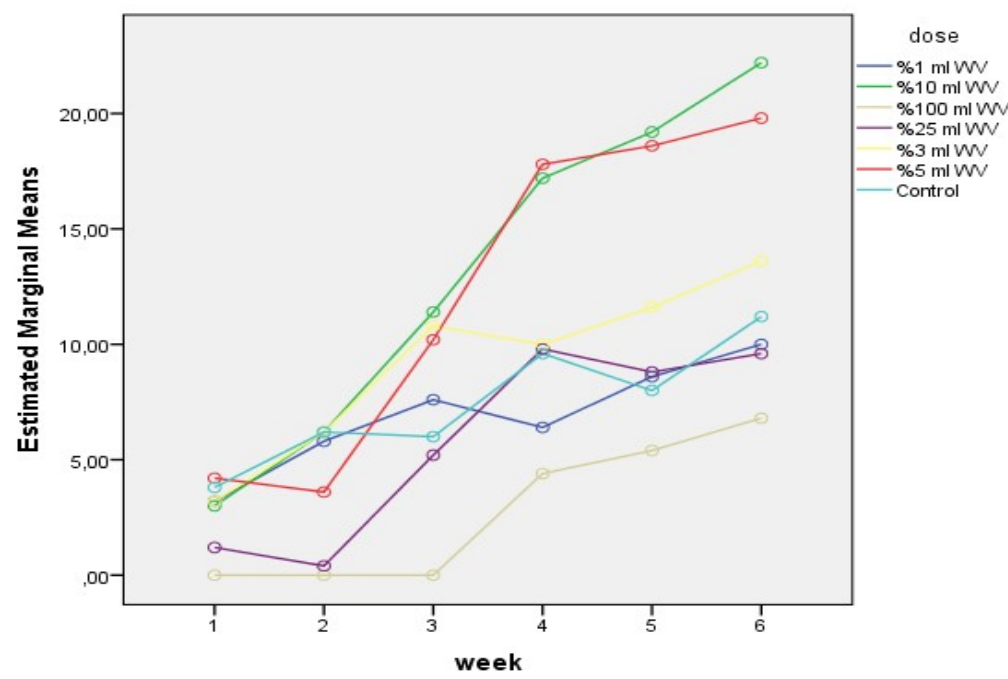

Figure 5. Profile analysis for investigating the effect of $W V(B)$ on weekly number of worms based on doses

Table 2. Descriptive statistics for number of worms

\begin{tabular}{|c|c|c|c|c|c|c|c|c|c|c|c|}
\hline Week & WV Dose & $\begin{array}{c}\text { Vinegar } \\
\text { type }\end{array}$ & Mean & $\begin{array}{c}\text { Std. } \\
\text { deviation }\end{array}$ & $\mathbf{N}$ & Week & WV Dose & $\begin{array}{c}\text { Vinegar } \\
\text { type }\end{array}$ & Mean & $\begin{array}{c}\text { Std. } \\
\text { deviation }\end{array}$ & $\mathbf{N}$ \\
\hline \multirow{9}{*}{$\stackrel{1}{3}$} & \multirow{3}{*}{$1 \%$} & A & 3.000 & 1.581 & 5 & \multirow{9}{*}{$\hat{3}$} & \multirow{3}{*}{$1 \%$} & A & 3.200 & 1.304 & 5 \\
\hline & & $\mathrm{B}$ & 3.200 & 1.304 & 5 & & & $\mathrm{~B}$ & 5.800 & 0.836 & 5 \\
\hline & & Total & 3.100 & 1.370 & 10 & & & Total & 4.500 & 1.716 & 10 \\
\hline & \multirow{3}{*}{$3 \%$} & A & 2.600 & 0.894 & 5 & & \multirow{3}{*}{$3 \%$} & A & 5.000 & 1.000 & 5 \\
\hline & & B & 3.200 & 0.447 & 5 & & & $\mathrm{~B}$ & 6.200 & 0.837 & 5 \\
\hline & & Total & 2.900 & 0.738 & 10 & & & Total & 5.600 & 1.075 & 10 \\
\hline & \multirow{3}{*}{$5 \%$} & A & 3.800 & 1.304 & 5 & & \multirow{3}{*}{$5 \%$} & A & 5.800 & 0.837 & 5 \\
\hline & & $\mathrm{B}$ & 4.200 & 0.447 & 5 & & & $\mathrm{~B}$ & 3.600 & 1.140 & 5 \\
\hline & & Total & 4.000 & 0.943 & 10 & & & Total & 4.700 & 1.494 & 10 \\
\hline
\end{tabular}




\begin{tabular}{|c|c|c|c|c|c|c|c|c|c|c|c|}
\hline & \multirow{3}{*}{$10 \%$} & $\mathrm{~A}$ & 3.400 & 1.673 & 5 & & \multirow{3}{*}{$10 \%$} & $\mathrm{~A}$ & 4.200 & 0.447 & 5 \\
\hline & & B & 3.000 & 1.414 & 5 & & & B & 6.200 & 1.095 & 5 \\
\hline & & Total & 3.200 & 1.476 & 10 & & & Total & 5.200 & 1.316 & 10 \\
\hline & \multirow{3}{*}{$25 \%$} & A & 2.000 & 1.414 & 5 & & \multirow{3}{*}{$25 \%$} & A & 1.000 & 1.000 & 5 \\
\hline & & $\mathrm{B}$ & 1.200 & 0.447 & 5 & & & $\mathrm{~B}$ & 0.400 & 0.548 & 5 \\
\hline & & Total & 1.600 & 1.075 & 10 & & & Total & 0.700 & 0.823 & 10 \\
\hline & \multirow{3}{*}{$100 \%$} & A & 0.000 & 0.000 & 5 & & \multirow{3}{*}{$100 \%$} & A & 0.000 & 0.000 & 5 \\
\hline & & B & 0.000 & 0.000 & 5 & & & B & \begin{tabular}{|l|l|}
0.000 \\
\end{tabular} & 0.000 & 5 \\
\hline & & Total & 0.000 & 0.000 & 10 & & & Total & 0.000 & 0.000 & 10 \\
\hline & \multirow{3}{*}{ Control } & A & 5.000 & 1.000 & 5 & & \multirow{3}{*}{ Control } & $\mathrm{A}$ & 5.200 & 0.447 & 5 \\
\hline & & $\mathrm{B}$ & 3.800 & 0.447 & 5 & & & $\mathrm{~B}$ & 6.200 & 1.304 & 5 \\
\hline & & Total & 4.400 & 0.966 & 10 & & & Total & 5.700 & 1.059 & 10 \\
\hline & \multirow{3}{*}{ Total } & A & 2.829 & 1.855 & 35 & & \multirow{3}{*}{ Total } & A & 3.486 & 2.215 & 35 \\
\hline & & B & 2.657 & 1.589 & 35 & & & $B$ & \begin{tabular}{|l|l}
4.057 \\
\end{tabular} & 2.754 & 35 \\
\hline & & Total & 2.743 & 1.717 & 70 & & & Total & 3.771 & 2.497 & 70 \\
\hline \multirow{24}{*}{$\stackrel{+}{3}$} & \multirow{3}{*}{$1 \%$} & A & 4.200 & 1.924 & 5 & & \multirow{3}{*}{$1 \%$} & A & 3.600 & 2.191 & 5 \\
\hline & & $B$ & 7.600 & 0.894 & 5 & & & B & 6.400 & 1.342 & 5 \\
\hline & & Total & 5.900 & 2.283 & 10 & & & Total & 5.000 & 2.261 & 10 \\
\hline & \multirow{3}{*}{$3 \%$} & A & 7.400 & 0.548 & 5 & & \multirow{3}{*}{$3 \%$} & $\bar{A}$ & 6.400 & $\begin{array}{c}0.894 \\
\end{array}$ & 5 \\
\hline & & $\mathrm{B}$ & 10.800 & 1.304 & 5 & & & B & 10.000 & 1.225 & 5 \\
\hline & & Total & 9.100 & 2.025 & 10 & & & Total & \begin{tabular}{|l|l|}
8.200 \\
\end{tabular} & 2.150 & 10 \\
\hline & \multirow{3}{*}{$5 \%$} & A & 15.200 & 1.789 & 5 & & \multirow{3}{*}{$5 \%$} & A & 13.600 & 3.050 & 5 \\
\hline & & B & 10.200 & 2.049 & 5 & & & B & 17.800 & 1.924 & 5 \\
\hline & & Total & 12.700 & 3.199 & 10 & & & Total & 15.700 & 3.268 & 10 \\
\hline & \multirow{3}{*}{$10 \%$} & $\mathrm{~A}$ & 4.600 & 1.517 & 5 & & \multirow{3}{*}{$10 \%$} & $\bar{A}$ & 7.800 & 3.493 & 5 \\
\hline & & B & 11.400 & 1.342 & 5 & & & B & 17.200 & 1.304 & 5 \\
\hline & & Total & 8.000 & 3.829 & 10 & $n$ & & Total & 12.500 & 5.543 & 10 \\
\hline & & $\mathrm{A}$ & 2.400 & 0.548 & 5 & 3 & & $\mathrm{~A}$ & 4.600 & 2.881 & 5 \\
\hline & $25 \%$ & B & 5.200 & 0.447 & 5 & & $25 \%$ & B & 9.800 & 3.962 & 5 \\
\hline & & Total & 3.800 & 1.549 & 10 & & & Total & 7.200 & 4.264 & 10 \\
\hline & & A & 0.000 & 0.000 & 5 & & & A & 0.000 & 0.000 & 5 \\
\hline & $100 \%$ & B & 0.000 & 0.000 & 5 & & $100 \%$ & B & 4.400 & 2.608 & 5 \\
\hline & & Total & 0.000 & 0.000 & 10 & & & Total & 2.200 & 2.898 & 10 \\
\hline & & A & 7.400 & 0.548 & 5 & & & A & 8.800 & 1.304 & 5 \\
\hline & Control & $\mathrm{B}$ & 6.000 & 0.707 & 5 & & Control & $\mathrm{B}$ & \begin{tabular}{|l|}
9.600 \\
\end{tabular} & 3.647 & 5 \\
\hline & & Total & 6.700 & 0.949 & 10 & & & Total & 9.200 & 2.616 & 10 \\
\hline & & $\mathrm{A}$ & 5.886 & 4.708 & 35 & & & $\mathrm{~A}$ & 6.400 & 4.558 & 35 \\
\hline & Total & B & 7.314 & 3.917 & 35 & & Total & B & 10.742 & 5.271 & 35 \\
\hline & & Total & 6.600 & 4.359 & 70 & & & Total & \begin{tabular}{|l|}
8.571 \\
\end{tabular} & 5.358 & 70 \\
\hline & & $\mathrm{A}$ & 2.600 & 3.578 & 5 & & & $\mathrm{~A}$ & \begin{tabular}{|l|}
3.400 \\
\end{tabular} & 3.507 & 5 \\
\hline & $1 \%$ & B & 8.600 & 1.817 & 5 & & $1 \%$ & B & 10.000 & 1.414 & 5 \\
\hline & & Total & 5.600 & 4.142 & 10 & & & Total & \begin{tabular}{|l|}
6.700 \\
\end{tabular} & 4.296 & 10 \\
\hline & & $\mathrm{A}$ & 19.000 & 1.871 & 5 & & & $\mathrm{~A}$ & 21.400 & 2.074 & 5 \\
\hline 0 & $3 \%$ & B & 11.600 & 1.517 & 5 & $\tau$ & $3 \%$ & $\mathrm{~B}$ & 13.600 & 2.191 & 5 \\
\hline 3 & & Total & 15.300 & 4.218 & 10 & 3 & & Total & 17.500 & 4.577 & 10 \\
\hline & & A & 18.800 & 2.588 & 5 & & & $\mathrm{~A}$ & 20.200 & 1.924 & 5 \\
\hline & $5 \%$ & B & 18.600 & 2.608 & 5 & & $5 \%$ & B & 19.800 & 3.033 & 5 \\
\hline & & Total & 18.700 & 2.452 & 10 & & & Total & 20.000 & 2.404 & 10 \\
\hline & $10 \%$ & $\mathrm{~A}$ & 12.600 & 4.278 & 5 & & $10 \%$ & $\mathrm{~A}$ & 15.200 & 2.280 & 5 \\
\hline
\end{tabular}




\begin{tabular}{|c|c|c|c|c|c|c|c|c|c|}
\hline & B & 19.200 & 1.643 & 5 & & B & 22.200 & 2.168 & 5 \\
\hline & Total & 15.900 & 4.630 & 10 & & Total & 18.700 & 4.244 & 10 \\
\hline \multirow{3}{*}{$25 \%$} & A & 3.400 & 2.408 & 5 & \multirow{3}{*}{$25 \%$} & A & 6.200 & 1.095 & 5 \\
\hline & B & 8.800 & 2.168 & 5 & & B & 9.600 & 2.191 & 5 \\
\hline & Total & 6.100 & 3.573 & 10 & & Total & \begin{tabular}{|l|l}
7.900 \\
\end{tabular} & 2.424 & 10 \\
\hline \multirow{3}{*}{$100 \%$} & A & 1.800 & 1.483 & 5 & \multirow{3}{*}{$100 \%$} & $\mathrm{~A}$ & 2.400 & 2.074 & 5 \\
\hline & B & 5.400 & 3.050 & 5 & & B & 6.800 & 3.899 & 5 \\
\hline & Total & 3.600 & 2.951 & 10 & & Total & \begin{tabular}{|l|}
4.600 \\
\end{tabular} & 3.748 & 10 \\
\hline \multirow{3}{*}{ Control } & $\mathrm{A}$ & 14.200 & 0.836 & 5 & \multirow{3}{*}{ Control } & $\mathrm{A}$ & 16.800 & 1.304 & 5 \\
\hline & $\mathrm{B}$ & 8.000 & 2.121 & 5 & & $\mathrm{~B}$ & 11.200 & 3.633 & 5 \\
\hline & Total & 11.100 & 3.604 & 10 & & Total & 14.000 & 3.916 & 10 \\
\hline \multirow{3}{*}{ Total } & $\mathrm{A}$ & 10.343 & 7.550 & 35 & \multirow{3}{*}{ Total } & $\mathrm{A}$ & 12.229 & 7.807 & 35 \\
\hline & $\mathrm{B}$ & 11.457 & 5.447 & 35 & & $\mathrm{~B}$ & 13.314 & 5.890 & 35 \\
\hline & Total & 10.900 & 6.559 & 70 & & Total & 12.771 & 6.887 & 70 \\
\hline
\end{tabular}

\section{Findings related to cocoon numbers}

Factorial ANOVA was used to analyze the data set. Results of ANOVA showed that the Dose $\times$ Vinegar type interaction is significant $(\mathrm{P}=0.00)$. Therefore, the effect of vinegar type on number of cocoons varied based on the doses (Table 3; Fig. 6). Upon comparison of WV (A) treatments with the controls, doses of 3\% and 5\% exerted positive effects on the number of cocoons, while treatments of $1 \%, 25 \%$ and $100 \%$ doses had negative effects. A smaller numbers of cocoons was produced under treatments with WV (B) compared to the controls (Table 3).

Table 3. Descriptive statistics and Tukey multiple comparison test for the number cocoons

\begin{tabular}{c|c|c|c|c|c}
\hline WV Dose & Vinegar type & Mean & Grouping & Std. error & N \\
\hline \multirow{3}{*}{$1 \%$} & A & 0.000 & $\mathrm{~b}$ & 0.000 & 5 \\
& $\mathrm{~B}$ & 1.600 & $\mathrm{a}$ & 1.342 & 5 \\
& Total & 0.800 & & 1.229 & 10 \\
\hline \multirow{3}{*}{$3 \%$} & $\mathrm{~A}$ & 4.000 & $\mathrm{a}$ & 0.707 & 5 \\
& $\mathrm{~B}$ & 0.400 & $\mathrm{~b}$ & 0.548 & 5 \\
& Total & 2.200 & & 1.989 & 10 \\
\hline \multirow{3}{*}{$5 \%$} & $\mathrm{~A}$ & 4.000 & $\mathrm{a}$ & 0.707 & 5 \\
& $\mathrm{~B}$ & 0.000 & $\mathrm{~b}$ & 0.000 & 5 \\
& Total & 2.000 & & 2.160 & 10 \\
\hline \multirow{3}{*}{$10 \%$} & $\mathrm{~A}$ & 3.200 & $\mathrm{a}$ & 1.304 & 5 \\
& $\mathrm{~B}$ & 1.600 & $\mathrm{a}$ & 0.548 & 5 \\
& Total & 2.400 & & 1.265 & 10 \\
\hline \multirow{3}{*}{$25 \%$} & A & 1.800 & $\mathrm{a}$ & 0.447 & 5 \\
& $\mathrm{~B}$ & 0.800 & $\mathrm{~b}$ & 0.837 & 5 \\
& Total & 1.300 & & 0.823 & 10 \\
\hline \multirow{3}{*}{$100 \%$} & A & 0.600 & $\mathrm{a}$ & 0.894 & 5 \\
& B & 0.000 & $\mathrm{a}$ & 0.000 & 5 \\
& Total & 0.300 & & 0.675 & 10 \\
\hline
\end{tabular}




\begin{tabular}{c|c|c|c|c|c}
\hline \multirow{3}{*}{ Control } & A & 3.200 & $\mathrm{a}$ & 0.836 & 5 \\
& $\mathrm{~B}$ & 2.600 & $\mathrm{~b}$ & 0.548 & 5 \\
& Total & 2.900 & & 0.738 & 10 \\
\hline \multirow{3}{*}{ Total } & $\mathrm{A}$ & 2.400 & $\mathrm{a}$ & 1.684 & 35 \\
& $\mathrm{~B}$ & 1.000 & $\mathrm{~b}$ & 1.111 & 35 \\
& Total & 1.700 & & 1.582 & 70 \\
\hline
\end{tabular}

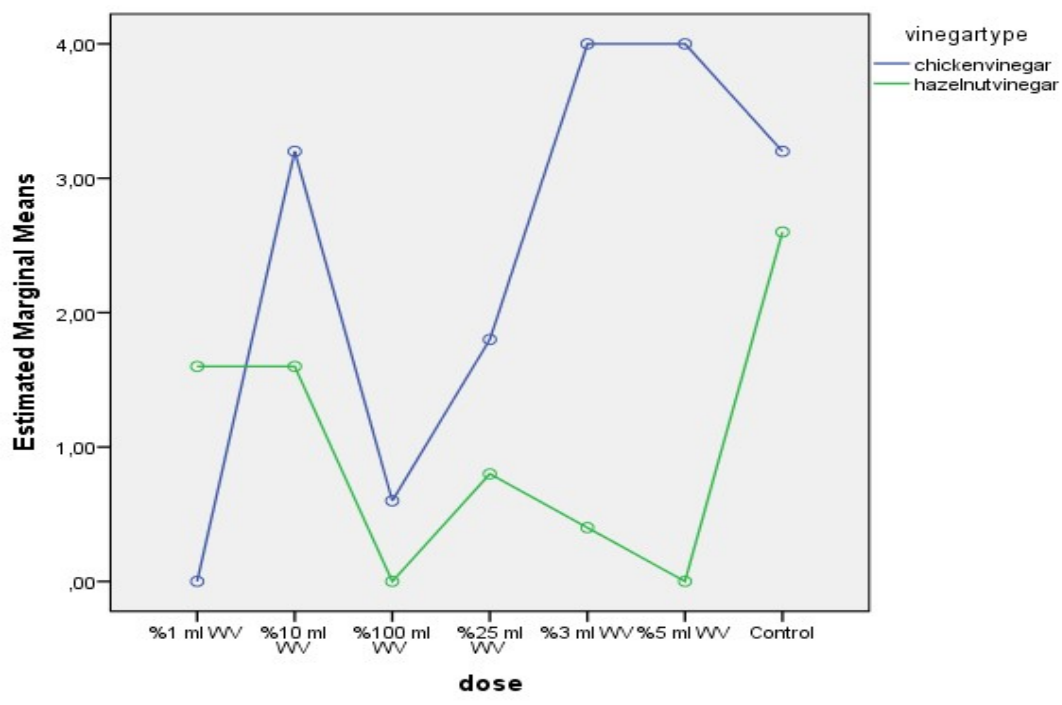

Figure 6. Changes in the number of cocoons based on dose and vinegar type

\section{Discussion}

Chemical pesticides and fertilizers used in agricultural activities pollute the environment, damage biodiversity and exert negative effects on human health. This situation can be eliminated through the use of alternative methods such as organic pesticides and manures. Wood vinegar (WV) is an organic product that can be degraded in a short time produced from plant and animal waste. Treatments with WV (A, B) have been found to have effects on the body weight, number of worms and number of cocoons depending on the type of vinegar, dose and the week of measurements. Of these vinegar types, WV (A) is thought to have exerted positive effects on the numbers of worms and cocoons particularly in doses of 3\% and 5\% (Table 3), while WV (B) has positive effects particularly on the number of worms in 3\%, 5\% and $10 \%$ doses (Table 2). These results are consistent with the findings of Cai et al. (2012) indicating that WV exerts positive effects on the development, lifetime and body size of worms. Furthermore, the fact that WV has positive effects on plants and other living beings in the soil is supported by the findings of Shi (2003), Jothityangkoon et al. (2008), Yin (2008), Rakmai (2009) and Cai et al. (2012). However, studies indicating the negative effects of a $1 \%$ dose on worms has been attributed to either an adverse factor in the test apparatus or to some toxic effect. Generally, the $100 \%$ dose and partially $25 \%$ dose of WV have been determined to exert negative effects on the biological parameters of worms. These results are supported by the findings of Inoue et al. (2000), Yatagai et al. (2002), Jothityangkoon et al. (2008), Yin (2008), Baimark and Niamsa (2009), 
Velmurugan et al. (2009), Chalermsan and Peerapan (2009), Diba et al. (2009), KiarieMakara et al. (2010), Pangnakorn et al. (2011), Wititsiri (2011), Eric et al. (2012), Oramahi and Yoshimura (2013), Hagner (2013), Namli et al. (2014), Duan et al. (2016), Yang et al. (2016), Koc et al. (2017), De Souza Araújo et al. (2018), Oramahi et al. (2018) and Chukeatirote et al. (2018), agreeing that WV can exert bactericide, fungicide, anti-termite and insecticide effects. However, it can easily be seen from the examination of figures and tables of these studies that the effect of types and doses of WV differ weekly. It is estimated that this can be attributed to the statements of Kim et al. (2008) and Jothityangkoon et al. (2008) in their respective studies, stating that vinegar consists of water-soluble substances exceeding 200 in number and the main components are acetic acid paired with organic acids, phenolic acids, alkanes, alcohol and esters.

\section{Conclusion}

As a result of this study we can conclude that types and doses of wood vinegar can have positive effects on certain parameters of Eisenia foetida. It is thought that the 3\% and $5 \%$ doses in particular exert positive effects on worms, while the dose of $100 \%$ has toxic effects. Vinegar types with all their characteristics may have a potential use in agriculture, or more precisely in organic agriculture and worm manure production.

Acknowledgements. I hereby present my great appreciations to Prof. Dr. Mehmet MENDEŞ for his help in the interpretations of the statistical analyses of this study and to Science Expert Birgül ILIKHAN for her help in laboratory works.

\section{REFERENCES}

[1] Baimark, Y., Niamsa, Y. (2009): Study on wood vinegars for use as coagulating and antifungal agents on the production of natural rubber sheets. - Biomass and Bioenergy 33(6-7): 994-998.

[2] Bustos-Obregón, E., Goicochea, R. I. (2002): Pesticide soil contamination mainly affects earthworm male reproductive parameters. - Asian Journal of Andrology 4(3): 195-200.

[3] Cai, K., Jiang, S., Renc, C., He, Y. (2012): Significant damage-rescuing effects of wood vinegar extract in living Caenorhabditis elegans under oxidative stress. - Journal of the Science of Food and Agriculture 92(1): 29-36.

[4] Chalermsan, Y., Peerapan, S. (2009): Wood vinegar: by-product from rural charcoal kiln and its role in plant protection. - Asian Journal of Food and Agro-Industry 2009(Special Issue): 189-195.

[5] Chukeatirote, E., Jenjai, N. (2018): Antimicrobial activity of wood vinegar from Dimocarpus longan. - EnvironmentAsia 11(3): 161-169.

[6] De Souza Araújo, E., Pimenta, A. S., Feijó, F. M. C., Castro, R. V. O., Fasciotti, M., Monteiro, T. V. C., De Lima, K. M. G. (2018): Antibacterial and antifungal activities of pyroligneous acid from wood of Eucalyptus urograndis and Mimosa tenuiflora. - Journal of Applied Microbiology 124(1): 85-96.

[7] Diba, F., Oramahi, H. A., Wahdina (2009): Antitermitic activity of wood vinegar and its components. - The First International Symposium of Indonesian Wood Research Society. 2nd-3rd November 2009, Bogor, Indonesia. 
[8] Duan, X., Wang, H., Liu, Z., Feng, C., Cui, Y. (2016): Antibacterial activities of wood vinegar from agricultural and forestry wastes dry distillation products. - Southwest China Journal of Agricultural Sciences 29(2): 425-429.

[9] Edwards, C. A., Bohlen, P. J. (1992): The Effects of Toxic Chemicals on Earthworms, in Reviews of Environmental Contamination and Toxicology. - Springer, New York, pp. 23-99.

[10] Eric, W., Chan, C., Fong, C. H., Kang, K. X., Chong, H. H. (2012): Potent antibacterial activity of wood vinegar from Matang mangroves, Malaysia. - ISME/GLOMIS Electronic Journal 10: 4.

[11] Gunadi, B., Edwards, C. A., Blount, C. (2003): The influence of different moisture levels on the growth, fecundity and survival of Eisenia fetida (Savigny) in cattle and pig manure solids. - European Journal of Soil Biology 39(1): 19-24.

[12] Hagner, M. (2013): Potential of the slow pyrolysis products birch tar oil, wood vinegar and biochar in sustainable plant protection - pesticidal effects, soil improvement and environmental risks. - Doctoral Dissertation, Department of Environmental Sciences Faculty of Biological and Environmental Sciences, University of Helsinki, Finland.

[13] Inoue, S., Hata, T., Imamura, Y., Meier, D. (2000): Components and anti-fungal efficiency of wood-vinegar-liquor prepared under different carbonization conditions. Wood research: Bulletin of the Wood Research Institute Kyoto University 87: 34-36.

[14] Jothityangkoon, D., Koolachart, R., Wanapat, S., Wongkaew, S., Jogloy, S. (2008): Using wood vinegar in enhancing peanut yield and in controlling the contamination of aflatoxin producing fungus. - International Crop Science 4: 253-253.

[15] Kim, D. H., Seo, H. E., Lee, S., Lee, K. (2008): Effects of wood vinegar mixted with insecticides on the mortalities of Nilaparvata lugens and Laodelphax striatellus (Homoptera: Delphacidae). - Animal Cells and Systems 12(1): 47-52.

[16] Kiarie-Makara, M. W., Yoon, H., Lee, D. (2010): Repellent efficacy of wood vinegar against Culex pipiens pallens and Aedes togoi (Diptera: Culicidae) under laboratory and semi-field conditions. - Entomological Research 40(2): 97-103.

[17] Koç, İ., Yardım, E. N., Yıldız, Ş. (2017): Antifungal effects of wood vinegar, derived from Broyler chicken manure, on microfungi under in-vitro conditions. - Yuzuncu Y1l University Journal of Agricultural Sciences 27(4): 516-520.

[18] Koç, İ., Yıldız, Ş., Yardım, E. N. (2018): The efficacy of wood vinegar and pesticides on soil nematodes in wheat agroecosystems. - UGAP 2018-1st International GAP Agriculture and Livestock Congress, pp. 330-330.

[19] Mendeş, M. (2012): Uygulamalı bilimler için istatistik ve araştırma yöntemleri. - Kriter Yayınevi, İstanbul.

[20] Mendeş, M., Dinçer, E., Arslan, E. (2007): Profile analysis and growth curve for body mass index of broiler chickens reared under different feed restrictions in early age. Archiv für Tierzucht 50(4): 403-411.

[21] Mu, J., Uehara, T., Furuno, T. (2003): Effect of bamboo vinegar on regulation of germination and radicle growth of seed plants. - Journal of Wood Science 49(3): 262270.

[22] Naml1, A., Akça, M. O., Turgay, E. B., Soba, M. R. (2014): Investigation of potential agricultural use of wood vinegar. - Soil Water Journal 3(1): 44-52.

[23] Oramahi, H. A., Yoshimura, T. (2013): Antifungal and antitermitic activities of wood vinegar from Vitex pubescens vahl. - Journal of Wood Science 59(4): 344-350.

[24] Oramahi, H. A., Yoshimura, T., Diba, F., Setyawati, D. (2018): Antifungal and antitermitic activities of wood vinegar from oil palm trunk. - Journal of Wood Science 64(3): 311-317.

[25] Pangnakorn, U., Kanlaya, S., Kuntha, C. (2011): Efficiency of wood vinegar and extracts from some medicinal plants on insect control. - Advances in Environmental Biology 5(2): 477-482. 
[26] Rakmai, J. (2009): Chemical determinations, antimicrobial and antioxidant activities of thai wood vinegars. - Master Dissertation, Prience of Songkla University, Thailand.

[27] Shi, Z. Y. (2003): The effects of wood vinegar on soil microorganisms and growth of vegetable seedlings. - Master Dissertation, China Agricultural University, China.

[28] Tiilikkala, K., Fagernäs, L., Tiilikkala, J. (2010): History and use of wood pyrolysis liquids as biocide and plant protection product history and use of wood pyrolysis liquids as biocide and plant protection product. - The Open Agriculture Journal 4(1): 111-118.

[29] Velmurugan, N., Han, S. S., Lee, Y. S. (2009): Antifungal activity of neutralized wood vinegar with water extracts of Pinus densiflora and Quercus serrata Saw Dusts. International Journal of Environmental Research 3(2): 167-176.

[30] Winer, B. J., Brown, D. R., Michels, K. M. (1971): Statistical Principles in Experimental Design. Vol. 2. - McGraw-Hill, New York.

[31] Wititsiri, S. (2011): Production of wood vinegars from coconut shells and additional materials for control of termite workers, Odontotermes sp. and striped mealy bugs, Ferrisia virgata. - Songklanakarin Journal of Science and Technology 33(3): 349-354.

[32] Yang, J. F., Yang, C. H., Liang, M. T., Gao, Z. J., Wu, Y. W., Chuang, L. Y. (2016): Chemical composition, antioxidant, and antibacterial activity of wood vinegar from Litchi chinensis. - Molecules 21(9): 1150.

[33] Yatagai, M., Nishimoto, M., Hori, K., Ohira, T., Shibata, A. (2002): Termiticidal activity of wood vinegar, its components and their homologues. - Journal of Wood Science 48(4): 338-342.

[34] Yasmin, S., D'Souza, D. (2010): Effects of pesticides on the growth and reproduction of earthworm: a review. - Applied and Environmental Soil Science. http://dx.doi.org/10.1155/2010/678360.

[35] Yin, A. L. (2008): Isolation and characterization of antioxidant compounds from pyroligneous acid of Rhizophora apiculata. - Doctoral Dissertation, Sains University, Malaysia. 